\title{
Quantitative Crack Analysis using Indirect Neutron Radiography and Neutron Activation Analysis with Contrast Enhancement Agents
}

\author{
Russell Jarmer ${ }^{1, \mathrm{a}}$, Dr. Jeffrey King ${ }^{1, \mathrm{~b},{ }^{,}, \text {Dr. Aaron Craft }}{ }^{2, \mathrm{c}}$, Dr. Robert O’Brien ${ }^{2, \mathrm{~d}}$ \\ ${ }^{1}$ Colorado School of Mines 1500 Illinois St. Golden, CO 80401 United States of America \\ ${ }^{2}$ Idaho National Laboratory 1955 N. Fremont Ave. Idaho Falls, ID 83415 United States of \\ America
}

ajarmer@mymail.mines.edu ${ }^{\mathrm{b}}$ kingjc@mines.edu ${ }^{\mathrm{c}}$ aaron.craft@inl.gov ${ }^{\mathrm{d}}$ robert.OBrien@inl.gov

\begin{abstract}
Keywords: Indirect Neutron Radiography, Contrast Agents, Quantitative Analysis, Neutron Activation Analysis
\end{abstract}

\begin{abstract}
This paper presents an analysis of infiltration and washing methods to develop a quantitative metric for crack features using indirect neutron radiography and neutron activation analysis. A gadolinium contrast agent enhances the visibility of crack features in digitally processed neutron radiographs. Neutron activation analysis of crack infiltrants using dysprosium as a surrogate for gadolinium directly measures the amount of infiltrant in crack features.
\end{abstract}

\section{Introduction}

Indirect neutron radiography is a valuable tool for the non-destructive examination of irradiated nuclear fuel; however, the ability to detect fine cracks in cladding materials is generally constrained by the spatial and contrast resolution limits of the imaging technique. Infiltrating fine cracks with a contrast agent can enhance the ability to image these cracks through neutron radiography. Establishing a documented standard for quantitative analysis of crack structures using neutron radiography with contrast-enhancing infiltrants will provide a valuable tool for examining irradiated materials, particularly advanced cladding materials. Phase One of this project tested three gadolinium infiltrant solutions containing varying amounts of methanol and ammonium lauryl sulfate to quantify the effect of lowering the surface tension of the infiltrant to enhance the ability of the infiltrant to penetrate small cracks. Scanning and digitally processing the resulting radiographic images provided a quantitative measurement of the crack density, extent of infiltrant penetration, and the effectiveness of washing to remove the residual gadolinium. Neutron Activation Analysis (NAA) of a dysprosium based infiltrant solution in Phase Two of the project directly measured the amount of infiltrant in the crack features to provide a comparison to the image-based crack densities in Phase One.

Image contrast is a primary limitation to crack detection through radiography. When filled with air, the cracks are difficult to distinguish from the surrounding material, as the difference between the neutron attenuation by the air and the neutron attenuation by the surrounding material is small. Increasing the imaging time can increase the contrast between the crack and the parent material. Alternatively, filling the crack with a highly attenuating contrast agent increases the contrast between the crack and the surrounding material without increasing imaging time [1].

A contrast agent for neutron radiography must provide a large neutron cross section, and must be easy to prepare into an infiltrant solution. Gadolinium (III) nitrate meets both requirements, as gadolinium provides the largest thermal neutron cross-section of any naturally occurring element [2], and gadolinium nitrate is readily soluble in water.

(c) (1) Content from this work may be used under the terms of the Creative Commons Attribution 3.0 license. Any further distribution of this work must maintain attribution to the author(s) and the title of the work, journal citation and DOI. Published under license by Materials Research Forum LLC. 
Phase One of this project investigated the ability of a gadolinium-based infiltrant to enhance the contrast of indirect neutron radiography images at the Neutron RADiography (NRAD) reactor at the Idaho National Laboratory. This reactor is a $250 \mathrm{~kW}$ TRIGA (Training, Research, Isotopes, General Atomics) reactor, and provides neutrons for two beamlines [3]. Two radiography stations at the NRAD allow for indirect neutron imaging using a cassette containing activation foils. Two activation foils, dysprosium for thermal neutrons and indium for epithermal neutrons, capture the neutrons that pass through a sample placed in the neutron beam in front of the cassette. The neutron attenuation by the materials in the sample creates an activated map of neutron intensity in the activation foils. After a sufficient irradiation time, placing the activation foil in contact with photographic film allows radiation produced by the decay of the activated atoms in the foil to expose the film. Developing the exposed film produces a radiographic image of the sample.

Phase Two of this project test the ability to infiltrate and remove contrast agent using Neutron Activation Analysis (NAA). NAA works by exposing a sample to a neutron field; materials within the sample absorb neutrons, creating new isotopes, some of which undergo radioactive decay. A High Purity Germanium (HPGe) detector counts gamma radiation emitted by these decays and bins the results by energy. These results determine the composition of the sample due to charactersitic decays of isotopes. Additionally, the number of decays of a particular isotope can be used to determine the mass of the parent isotope present in the sample [4].

\section{Methodology}

Neutron Radiography Methods. A set of ten numbered aluminum test blocks made following ASTM standards provided a documented standard object for investigating the ability of potential contrast agents to infiltrate cracks and appear in subsequent radiographs. Conventional dye penetrant testing provided a useful comparison to the imaging of cracks via neutron radiography. A 15 minute dwell period after application of the dye penetrant allowed full penetration of crack features. A white developing agent sprayed on the surface of the block pulled the dye out of the cracks to reveal the crack patterns. Optical photographs of the cracks, taken immediately after developer application and after a two minute development period, provided the reference images for the neutron radiography experiments (Fig. 1). Dye penetrant testing revealed no cracking in Blocks 4 and 8, excluding those blocks from further testing.

Table 1 lists the combinations of contrast agents and solvents used in infiltrant and washing solutions used in this experiment. The different solutions test how lowering the viscosity and surface tension of the infiltrant solutions improved the ability of the infiltrant to penetrate cracks under vacuum.

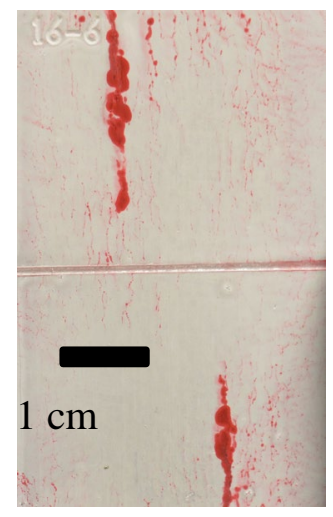

Figure 1. Reference image of cracks in Block 6 revealed by dye penetrant testing. 
Table 2 details the infiltration and wash steps for each block. The steps in each test sequence of Phase One proceeded in the order presented in the first column of Table 2 (i.e. Wash 2 and Wash 3 (Steps 4 and 5) were followed by Infiltration 3 (Step 6)). The second and third columns in Table 2 give the step name and step ID (used in Figures 3-6). The next four columns list the solution from Table 1 used to perform the process listed in the final column for that step, as a function of the block being treated. The project obtained new radiographs of the blocks after each step in Table 2.

Table 1. Phase One (neutron radiography) infiltration and washing solutions.

\begin{tabular}{lcl}
\hline & Contrast Agent & Solvent Composition \\
\hline Solution 1 & $0.3 \mathrm{M} \mathrm{Gd}\left(\mathrm{NO}_{3}\right)_{3}$ & Water \\
Solution 2 & $0.3 \mathrm{M} \mathrm{Gd}\left(\mathrm{NO}_{3}\right)_{3}$ & $50 \%$ water/50\% methanol (vol\%) \\
Solution 3 & $0.3 \mathrm{M} \mathrm{Gd}\left(\mathrm{NO}_{3}\right)_{3}$ & $\begin{array}{l}50 \% \text { water/50\% methanol (vol\%) with } 5 \mathrm{wt} \% \text { ammonium } \\
\text { lauryl sulfate }\end{array}$ \\
Solution 4 & None & Water \\
Solution 5 & None & $50 \%$ water/50\% methanol (vol\%) \\
\hline
\end{tabular}

Table 2. Phase One (neutron radiography) infiltration and washing methods.

Solution (From Table 1)

Blocks Blocks Blocks Blocks*

Step Step Name ID $\quad 1 \& 2 \quad 6 \& 9 \quad 5 \& 7 \quad 3 \& 10 \quad$ Process

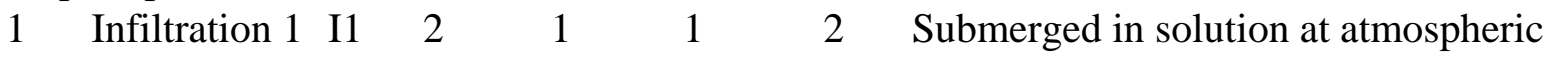

pressure for 5 minutes

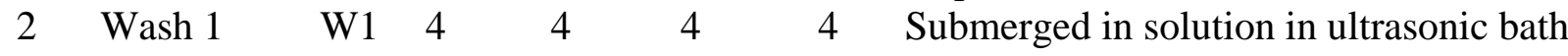
for 5 minutes followed by 5 minutes under -0.03 MPa vacuum

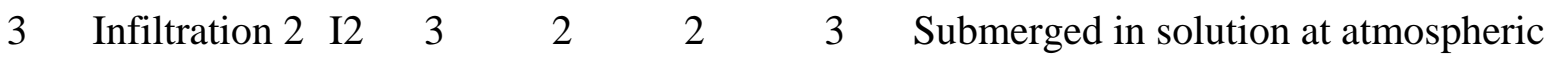

pressure for 15 minutes

$\begin{array}{lllllll}4 & \text { Wash } 2 & \text { W2 } & 5 & 5 & 5 & 4\end{array}$ Submerged in solution in ultrasonic bath

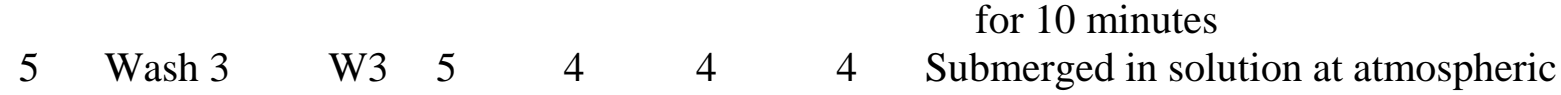
pressure for 15 minutes followed by 15 minutes under -0.03 MPa vacuum

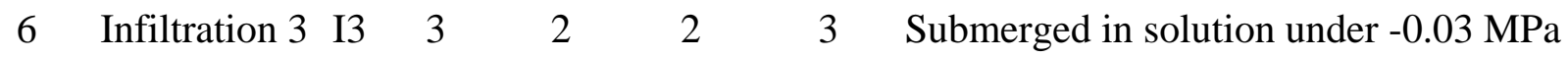
vacuum for 7.5 minutes, repeated $4 \mathrm{x}$

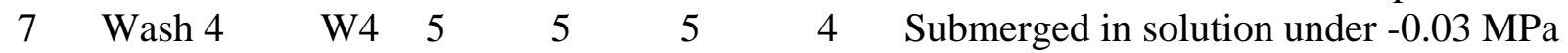
vacuum for 22.5 minutes then placed in an ultrasonic bath for 5 minutes, repeated 3 times

8 Wash $5 \quad$ W5 $4 \quad 4 \quad 4 \quad 4 \quad 4$

4 Submerged in solution and placed under $0.03 \mathrm{MPa}$ vacuum for 25 minutes. Then $3 x 5$ minute cycles under $-0.03 \mathrm{MPa}$ vacuum and 5 minutes in an ultrasonic bath

${ }^{*}$ Dye Penetrant testing did not reveal cracks in Blocks 4 and 8 
As an example, the infiltration and washing sequence for Block 6 started with an infiltration in a water-based solution of the contrast agent (Solution 1 in Table 1) at atmospheric pressure for five minutes. The next step was Wash 1, which put the block in a solution of clean water (Solution 4 in Table 1), and cleaned it in an ultrasonic bath for five minutes followed by five minutes at $-0.03 \mathrm{MPa}$ vacuum. Infiltration 2 then infiltrated Block 6 a second time; this step submerged the block at atmospheric pressure for 15 minutes in a solution of the contrast agent in a 50/50 vol\% mixture of water and methanol (Solution 2 in Table 1). Wash Step 2 used only a 10 minute ultrasonic bath using a cleaning solution of 50/50 vol\% water and methanol (Solution 4 in Table 1). This was followed by another wash step (Wash 3) that used clean water and submerged the sample for 15 minutes at atmospheric pressure then for 15 more minutes under Table 2 details the infiltration and wash steps for each block. The steps in each $-0.03 \mathrm{MPa}$ vacuum. Infiltration 3 again used Solution 2 in Table 1 (50/50 vol\% water and methanol mixture with a contrast agent), submerging the sample then subjecting it to $-0.03 \mathrm{MPa}$ vacuum for 7.5 minutes, after which the vacuum was released, then redrawn back to $-0.03 \mathrm{MPa}$. This was repeated for a total of four vacuum cycles. Wash 4 for Block 6 used a washing solution of 50/50 vol\% water and methanol to submerge the solution, followed by a vacuum of $-0.03 \mathrm{MPa}$ for 22.5 minutes and an ultrasonic bath for five minutes. The vacuum and ultrasonic cycles were then repeated 2 additional times. The final step for Block 6 (Wash 5 in Table 2) used clean water (Solution 4 in Table 1) and an initial vacuum of $-0.03 \mathrm{MPa}$ for 25 minutes followed by three cycles of five minutes under $-0.03 \mathrm{MPa}$ vacuum and five minutes in an ultrasonic bath.

The following process produced the neutron radiographs taken at the East Radiography station after each infiltration or wash step: After a 22 minute exposure of the samples in the beamline, the activation foils were placed in contact with the radiographic film overnight; then, the film was developed the next day by an automatic film processor. The crack metric is based on the images produced by the dysprosium foil.

Next, image processing of the digital scans of the film radiographs created a metric based on the percentage of black pixels in the selected crack boundary box. Fig. 2 shows a crack as it appears across each of the steps in the digital metric process. Step 1 took the cropped radiograph (Fig. 2a) and used Image J [5] to invert and shift the histogram to a constant value (Fig. 2b). Step 2 used a rolling ball algorithm to remove non-crack noise from the image (Fig. 2c), Step 3 applied a threshold to the crack features to make crack features black and the rest of the block white (Fig. 2d), and Step 4 isolated individual crack features in each block (Fig. 2e).

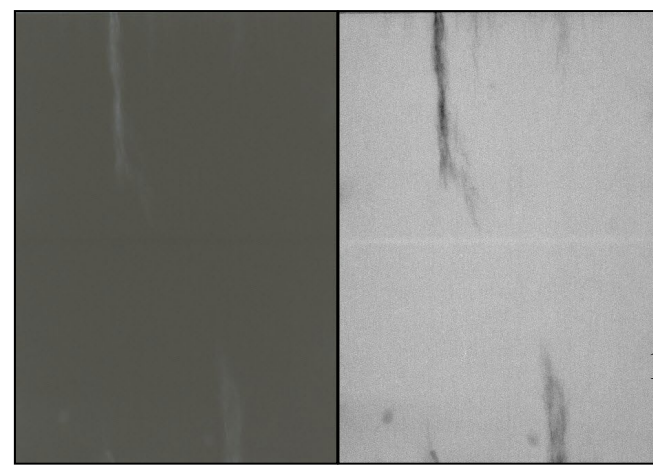

a) Initial radiograph. b) Normalized radiograph.

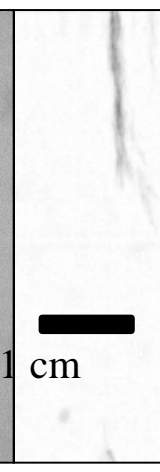

c) Subtracted background.

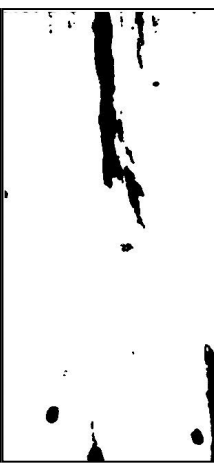

d) Threshold Image.

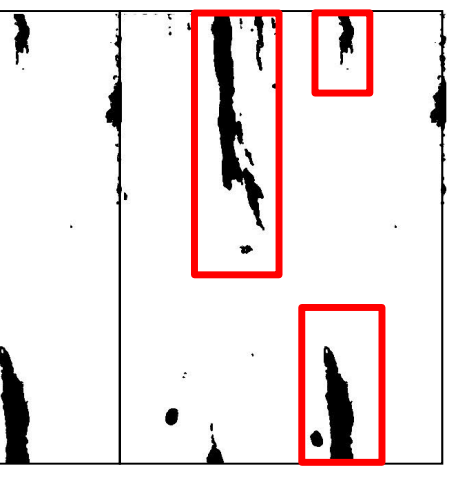

e) Isolated cracks.

Figure 2. Images produced by each step of digital metric process (Block 6). 
Neutron Activation Analysis Methods. Phase Two replaced the gadolinium contrast agent with dysprsoium as the primary neutron capture isotope of gadolinium creates a stable isotope, making NAA based on this isotope impractical. Dysprosium has similar chemistry to gadolinium, and dysprosium-164 has a substantial (2840 b) thermal neutron cross section, and the activation product dysprosium-165 has a 2.13 hour half-life [2,6]. The Geological Survey TRIGA Reactor (GSTR), a $1 \mathrm{MW}_{\text {th }}$ reactor, owned and operated by the United States Geological Survey, irradiated the samples for the NAA testing. Eq. 1 relates the count rate of an activation product to the number of parent isotope atoms.

$$
\frac{\mathrm{C}}{\varepsilon \mathrm{y}}=\frac{\mathrm{N}_{\mathrm{a}} \sigma \phi}{\lambda}\left[1-e^{-\lambda t_{i}}\right]\left[e^{-\lambda t_{d}}\right]\left[1-e^{-\lambda t_{c}}\right]
$$

where $\mathrm{C}$ is the number of gamma rays from the decay of the product isotope of interest recorded by the HPGe detector, $\varepsilon$ is the detector efficiency for the corresponding gamma ray energies, and $\mathrm{y}$ is the branching ratio of the decay of interest. $\mathrm{N}_{\mathrm{a}}$ is the number of atoms of the parent isotope present in the sample, $\sigma$ is the thermal neutron cross section for parent isotope, $\phi$ is the thermal neutron flux experienced by the sample during irradiation, and $\lambda$ is the decay constant of the product isotope. The times in the exponential terms are irradiation time $\left(\mathrm{t}_{\mathrm{i}}\right)$, decay time $\left(\mathrm{t}_{\mathrm{d}}\right)$ and count time $\left(\mathrm{t}_{\mathrm{c}}\right)$.

Phase Two included four new crack samples, made using ASTM procedures as a guideline, and a control sample. Table 3 describes the mass, composition, and size of the samples, as well as a description of the cracks in the samples. Axial cracks propagate along the direction of extrusion from the manufacturing process. Through cracks are observable on both the front and the back of the sample, while surface cracks appear on a single sample surface.

Table 3. Phase Two (NAA) sample details.

\begin{tabular}{|c|c|c|c|c|}
\hline Sample & Mass (g) & Composition & Sample Type & Crack Description \\
\hline 1 & 4.10 & Aluminum 2024 & 2” wide, $0.375 ”$ thick bar & Axial through crack \\
\hline 2 & 3.23 & Aluminum 2024 & 2" wide, 0.375 " thick bar & Axial through crack \\
\hline 3 & 6.61 & Aluminum 2024 & $\begin{array}{l}1.5 \text { " outer diameter pipe } \\
\text { with } 0.25 \text { " wall thickness }\end{array}$ & Axial surface crack \\
\hline 4 & 1.52 & Aluminum 6061 & $\begin{array}{l}1 \mathrm{~cm} \text { outer diameter tube } \\
\text { with } 1 \mathrm{~mm} \text { wall thickness }\end{array}$ & Axial surface crack \\
\hline control & 3.17 & Aluminum 2024 & 2” wide, 0.375 ” thick bar & No crack \\
\hline
\end{tabular}

Table 4. Phase Two (NAA) infiltration and washing methods.

\begin{tabular}{|c|c|c|}
\hline Process Step & Method & Solution Composition \\
\hline Infiltration & $\begin{array}{l}\text { Submerged in solution at atmospheric } \\
\text { pressure for } 5 \text { minutes }\end{array}$ & $\begin{array}{l}0.3 \mathrm{M} \mathrm{Dy}\left(\mathrm{NO}_{3}\right)_{3} \text { in de-ionized } \\
\text { water }\end{array}$ \\
\hline Wash 1 & Surface scrub & De-ionized water \\
\hline Wash 2 & $\begin{array}{l}\text { Submerged in solution in ultrasonic bath for } \\
30 \text { minutes, repeated once }\end{array}$ & De-ionized water \\
\hline Wash 3 & $\begin{array}{l}\text { Submerged in solution in ultrasonic bath for } \\
10 \text { minutes, repeated four times }\end{array}$ & De-ionized water \\
\hline
\end{tabular}


Table 4 shows the methods of infiltration and washing as well as the composition of the solutions used in Phase Two. The infiltration step submerged the five samples in the infiltrant solution at atmospheric pressure for five minutes. Wash 1 attempted to remove residual infiltrant from the surfaces, while Wash 2 and 3 submerged the samples in de-ionized water with multiple cycles in an ultrasonic bath to remove deposited infiltrant from the cracks. Neutron Activation Analysis determined the mass of infiltrant remaining in the crack sample after each process step in Table 5, based on Eq. 1. Solving Eq. 1 also determined the neutron flux in each capsule during each irradiation based on the mass and resulting activity of a gold wire flux monitor included in each sample capsule.

Table 5. Phase Two (NAA) irradiation details.

\begin{tabular}{|c|c|c|c|c|c|}
\hline $\begin{array}{l}\text { Process } \\
\text { Step }\end{array}$ & Capsule & Samples & $\begin{array}{l}\text { Flux Monitor } \\
\text { Mass (g) }\end{array}$ & $\begin{array}{l}\text { Irradiation } \\
\text { Time (s) }\end{array}$ & $\begin{array}{l}\text { Calculated Flux } \\
\text { (neutron } / \mathrm{cm}^{2} \mathrm{~s} \text { ) }\end{array}$ \\
\hline \multirow[t]{3}{*}{ Infiltration } & 1 & 1,4 & 0.1210 & 5.1 & $1.42 \times 10^{12}$ \\
\hline & 2 & 2,3 & 0.1206 & 5.1 & $1.39 \times 10^{12}$ \\
\hline & 3 & control & 0.1236 & 6.3 & $1.33 \times 10^{12}$ \\
\hline \multirow[t]{2}{*}{ Wash 1} & 1 & 2,3 & 0.1232 & 8.1 & $1.30 \times 10^{12}$ \\
\hline & 2 & 1,4 , control & 0.1237 & 8.1 & $1.34 \times 10^{12}$ \\
\hline \multirow[t]{2}{*}{ Wash 2} & 1 & 1,4, control & 0.1246 & 8.5 & $1.36 \times 10^{12}$ \\
\hline & 2 & 2,3 & 0.1235 & 8.5 & $1.39 \times 10^{12}$ \\
\hline \multirow[t]{2}{*}{ Wash 3} & 1 & 1,4 , control & 0.0071 & 8.1 & $1.16 \times 10^{12}$ \\
\hline & 2 & 2,3 & 0.0080 & 8.1 & $1.17 \times 10^{12}$ \\
\hline
\end{tabular}

\section{Results and Analysis}

Phase One demonstrated that infiltrating crack features with gadolinium enhanced the contrast of the features in resulting radiographs, allowing the development of a digital crack measurement. Phase Two directly measured the mass of a dysprosium infiltrant crack features using NAA. Changes in the size of the measured crack features between successive infiltration and washing steps in Phase One determined the effectiveness of each processing step. Changes in the mass of infiltrant measured between each step in Phase Two provided a check on the validity of the Phase One image-based metric.

Neutron Radiography Results. The Phase One image-based metric tests included eight blocks, yielding 21 distinct cracks. Each infiltration or washing step produced an image-based crack measurement as shown in Fig. 2. Figs. 3-6 present the percent change in the measured crack area as a function of the infiltration and washing steps described in Table 2. 


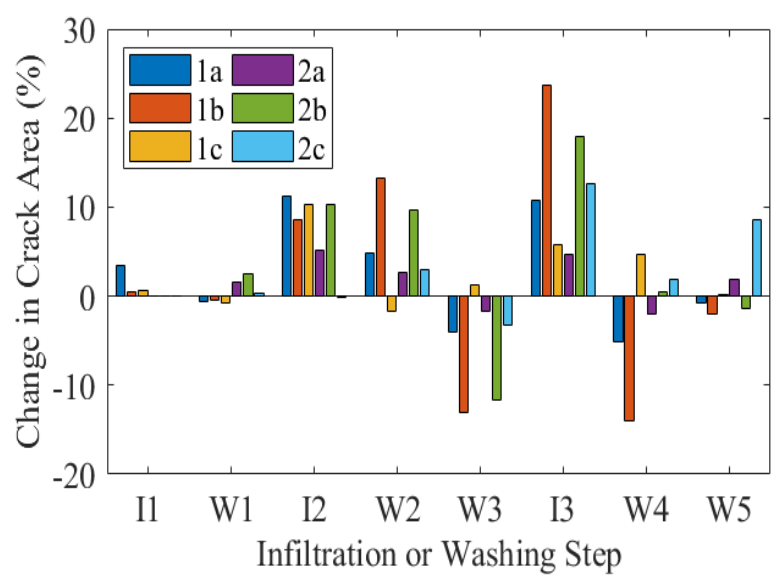

Figure 3. Changes in measured crack area as a function of processing step for blocks 1\&2 in Table 2.

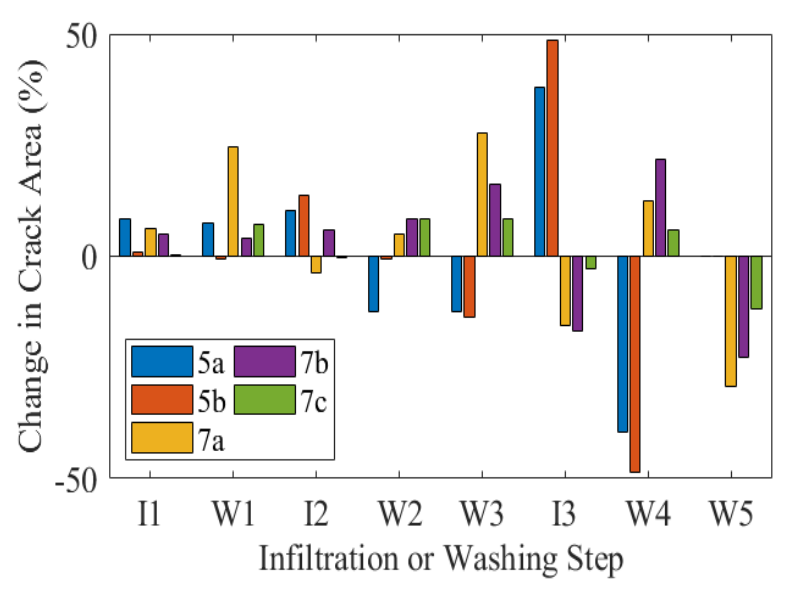

Figure 5. Changes in measured crack area as a function of processing step for blocks $5 \& 7$ in Table 2.

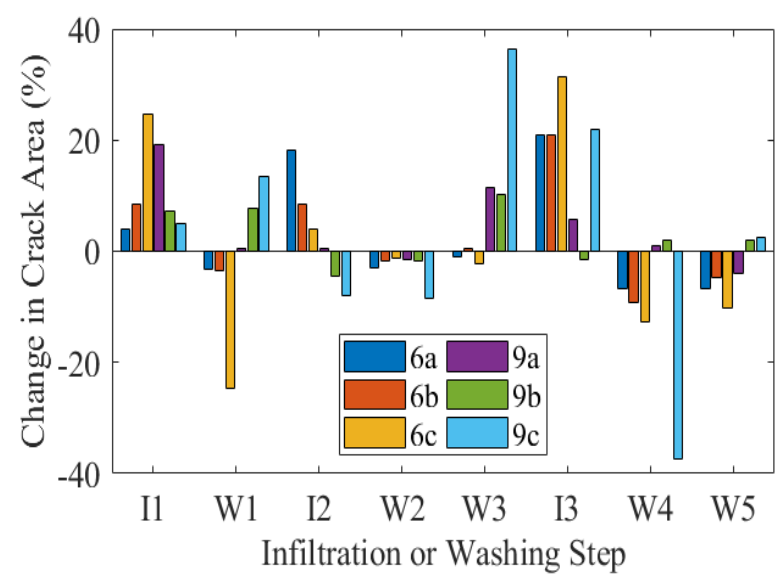

Figure 4. Changes in measured crack area as a function of processing step for blocks $6 \& 9$ in Table 2.

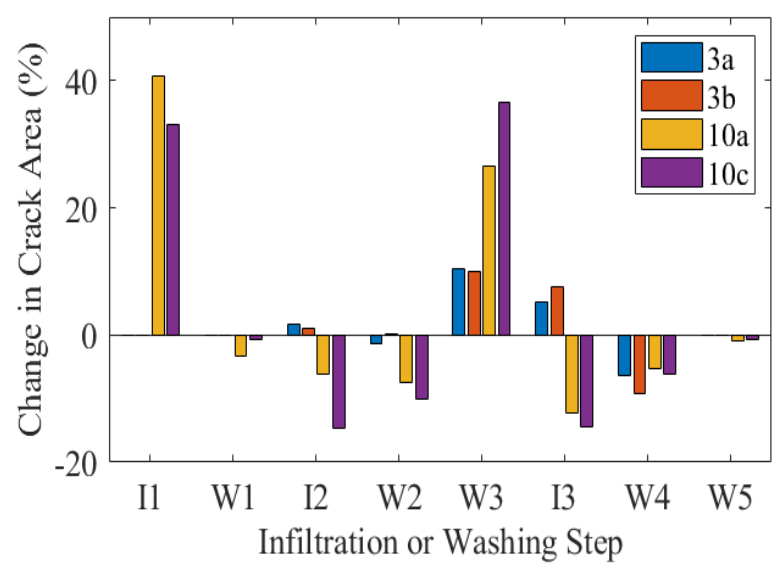

Figure 6. Changes in measured crack area as a function of processing step for blocks 3\&10 in Table 2.

In general, infiltration steps that included multiple vacuum phases (Step I3 in Figs. 3-5) resulted in the largest increases in measured crack area, indicating that multiple vacuum phases were particularly effective at depositing gadolinium in cracks. This trend was not observed in Blocks 3 and 10 in Fig. 6, because Step W3 may have fully infiltrated the crack, preventing the subsequent infiltration step (I3) from further increasing the amount of gadolinium in the cracks. Infiltration Step 3, applied to Blocks 5, 7, 6, and 9 (Figs. 4 and 5), resulted in slightly more consistent infiltration than the same process applied to Blocks 1, 2, 3, and 10 (Figs. 3 and 6), indicating that adding a surfactant (ammonium lauryl sulfate) to the solution did not increase the amount of gadolinium deposited in the cracks. Wash Step 4 in Figs. 3-6, generally removed the largest amount of gadolinium, indicating that multiple cycles of vacuum and ultrasonic cleaning are the most effective way to remove the contrast agent from the cracks.

Neutron Activation Analysis Results. Figs. 7a and 7b show the mass of dysprosium in the crack samples measured by NAA after the initial infiltration (Fig. 7a) and the change in the mass indicated by NAA after each washing step in Table 4 (Fig. 7b). The cracks in Samples 1, 2, and 3 took up more dysprosium during the Infiltration Step than the crack in Sample 4 (which was also 
not indicated by dye penetrant testing). The mass of dysprosium indicated in the Control Sample in Figs. 7a and 7b was most likely trace infiltrant on the surface of the sample. Thus, the first wash step in Phase Two focused only on removing residual infiltrant from sample surfaces.

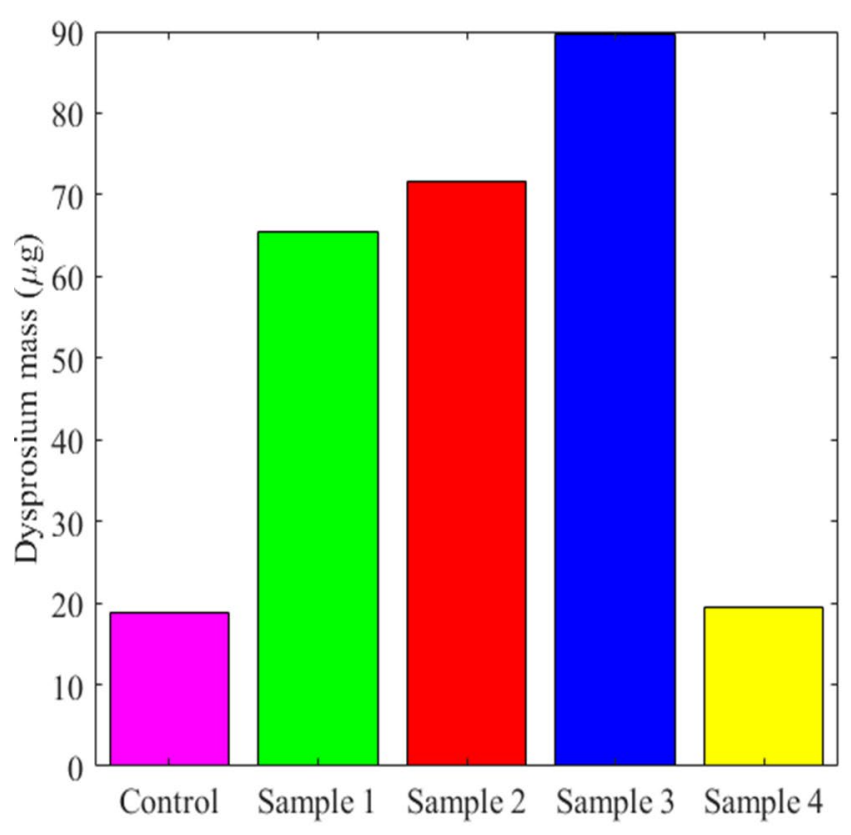

a) Mass of dysprosium indicated by NAA after Step 1 in Table 4.

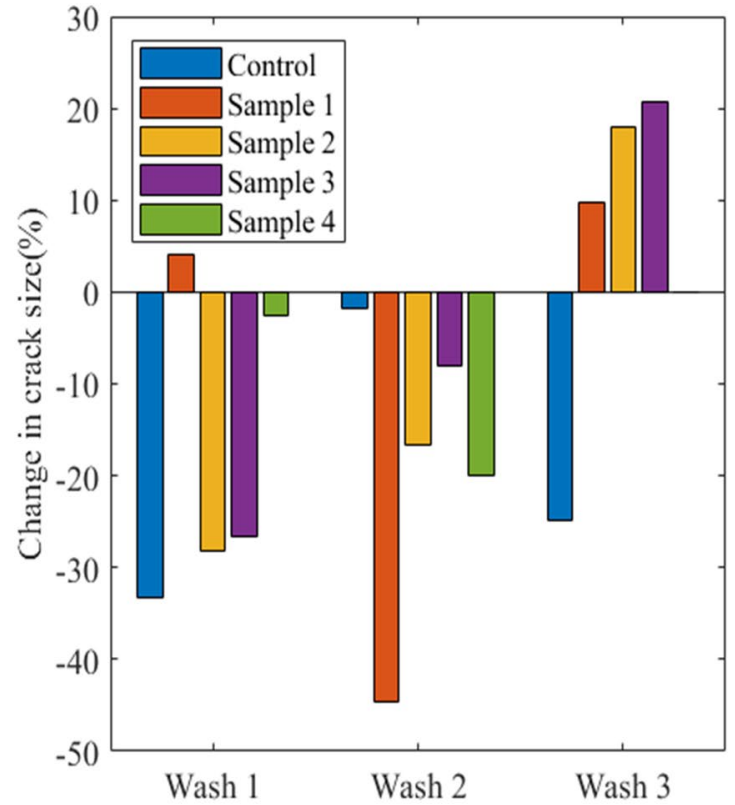

b) Percent mass of dysprosium removed by each wash Step in Table 4.

Figure 7. Neutron Activation Analysis metric results.

Wash Step 1 in Table 4 removed $~ 30 \%$ of the dysprosium from the surface of the uncracked sample (see Fig. 7b). Therefore, a thorough surface washing was necessary to remove the residual infiltrant from the non-cracked portions of the sample after each infiltration step. Ideally, a thorough surface cleaning of residual infiltrant from an uncracked sample would remove $100 \%$ of the residual infiltrant, indicating that the control sample may contain extremely fine cracks or pores that the infiltrant was able to penetrate.

Wash Step 2 in Table 4 removed $20 \%$ more dysprosium than Wash Step 3, indicating that longer cycles in the ultrasonic are more important than the number of cycles (see Fig. 7b). Since the washing solution was not changed between ultrasonic cycles, the additional cycles in Wash 3 may have deposited additional infiltrant in the cracks.

\section{Summary and Conclusions}

Phase One of this project tested the viability of a quantitative crack metric based on the analysis of digital scans of neutron radiographs of cracks infiltrated with gadolinium (III) nitrate. This phase also tested methods of infiltrating and washing the gadolinium contrast agent to/from cracks in standard aluminum test blocks. Gadolinium nitrate is an effective contrast agent, and image processing of the resulting radiographs produced a useable metric; however, the results from the washing methods were inconsistent and sometimes indicated increased crack extent after washing. Phase Two considered Neutron Activation Analysis as a secondary metric to directly measure the mass of an infiltrant present in crack samples. This metric also indicated inconsistencies in the effectiveness of the washing steps. Developing an effective and consistent washing process will be necessary for the use of neutron imaging with a contrast enhancement agent as a pre-irradiation examination technique. 
Future work will refine the infiltration and washing methods and may involve solutions containing both radiography contrast and NAA agents which will allow a direct comparison of the digital image processing data with the mass calculations to validate the neutron image based crack metric. The characteristic gamma ray emitted by dysprosium-165 is very low energy (94.7 $\mathrm{KeV}$ ) and has a low branch frequency (3\% of decays), leading to longer irradiation and count times to yield good statistics. An isotope with multiple, higher energy, and more frequent decays, may reduce the uncertainty in the mass calculations as several data points would be available to independently calculate the mass of infiltrant in each crack, allowing shorter irradiation and count times. The shorter irradiation times would produce lower amounts of activated materials, meaning less dose to personnel handling the samples after irradiation. Shorter count times also reduce potential dose and increase the number of countable samples for a given period.

\section{References}

[1] Brenizer J.S., Hosticka B., Berger H., Gillies G.T., The Use of Contrast Agents to Enhance Crack Detection Via Neutron Radiography, NDT \& E International, 32 (1999) 37-42. https://doi.org/10.1016/S0963-8695(98)00024-3

[2] Information on https://www.ncnr.nist.gov/resources/n-lengths/

[3] Craft A.E, Wachs D.M., Okuniewski M.A., Chichester D.L., Williams W.J., Papaioannou G.C., Smolinski A.T., Neutron Radiography of Irradiated Nuclear Fuel at Idaho National Laboratory, Physics Procedia, 69 (2015) 483-490. https://doi.org/10.1016/j.phpro.2015.07.068

[4] Shulyakova O., Avtonomov P., Kornienko V., New Developments of Neutron Activation Analysis Applications, Procedia-Social and Behanvioral Sciences, 195 (2015) 2717-2725. https://doi.org/10.1016/j.sbspro.2015.06.380

[5] Rasband, W.S., ImageJ, U. S. National Institutes of Health, Bethesda, Maryland, USA, https://imagej.nih.gov/ij/, 1997-2016.

[6] Information on https://www.nndc.bnl.gov/chart/ 